\title{
Remuneração dos (as) professores (as) da educação básica em Mato Grosso: sua evolução no contexto da lei de carreira e os desafios da valorização do magistério
}

\author{
Josete Maria Cangussú Ribeiro ${ }^{1}$ \\ josemcr@terra.com.br
}

\author{
Regis Queiroz Gonçalves ${ }^{2}$ \\ regisqg@yahoo.com.br
}

\section{Resumo}

A Lei Complementar n ${ }^{\circ}$ 050, de 01 de outubro de 1998, criou a carreira dos Profissionais da Educação Básica no Estado de Mato Grosso. A sua implementação, nesse estado, pode ser auferida a partir do estudo da remuneração docente desde o período de sua criação até os dias atuais. $\mathrm{O}$ acompanhamento da evolução da remuneração, definida como subsídio, fixado em parcela única, praticado de acordo com as classes e níveis, elementos estruturantes da carreira, permite conhecer a estrutura e a dinâmica da carreira docente que, além dos (as) professores (as), engloba também os demais profissionais atuantes na Educação Básica. $\mathrm{O}$ estudo aqui apresentado refere-se ao cargo de professor (a) e demonstra, a partir de quadros, tabelas e gráficos, os coeficientes e os respectivos valores de subsídios (remuneração) operados para cada classe e o respectivo nível, de acordo com o tempo trabalhado. O nível inicial é base para definição do Piso Salarial, e serve como parâmetro para os demais incrementos inseridos no decorrer da carreira docente.

Palavras-chaves: carreira; classes; coeficientes; níveis; remuneração.

1 Mestre em Educação pela Universidade Federal do Rio Grande do Sul, Brasil (2002). Professor titular da Universidade do Estado de Mato Grosso, Brasil

2 Mestre em Estatística pela Universidade Federal de Minas Gerais, Brasil (2006). Professor da Universidade Estadual do Mato Grosso, Brasil 


\section{Introdução}

A Lei Complementar nº 050, de 01 de outubro de 1998, dispõe sobre a carreira dos Profissionais da Educação Básica do Estado de Mato Grosso. O seu cumprimento pode ser verificado a partir dos holerites mensais ou folha de pagamento onde consta a remuneração efetivada a partir de subsídios.

Este estudo tem como base, além da Lei Complementar de criação da carreira, as demais legislações complementares que fixam o subsídio dos Profissionais da Educação Básica do Poder Executivo Estadual, a cada ano, mediante negociações ocorridas entre o Governo e a categoria docente, representada por seus sindicatos. Demonstra a evolução dos subsídios, a partir da estrutura de carreira, organizada em classes e níveis. Os coeficientes estabelecidos para cada classe e nível, conforme a titulação dos profissionais e o seu tempo de enquadramento na carreira, empossados a partir de concurso público, são indicadores a partir dos quais se define a política de remuneração no decorrer do tempo trabalhado.

A remuneração do nível inicial define o piso salarial e constitui a base usada como fator para os acréscimos auferidos a cada três anos de efetivo exercício no cargo. O presente estudo permite, dessa forma, compreender que a estrutura de carreira e a sua efetiva implementação, aliadas a outros elementos das políticas educacionais como, por exemplo, a política de financiamento e a garantia do custo-aluno-qualidade, são elementos basilares capazes de viabilizar uma verdadeira valorização dos profissionais da educação e, consequentemente, a qualidade dos processos educacionais.

\section{A estrutura de carreira dos profissionais da educação no Estado de Mato Grosso}

Compreender a estrutura da Lei de Carreira dos Profissionais da Educação Básica da esfera estadual de ensino do Estado de Mato Grosso é um exercício fundamental para acompanhar a evolução da remuneração docente dessa categoria de profissionais. O nosso objetivo é demonstrar a remuneração praticada a partir do Plano de Cargos, Carreira e Subsídios 
aprovado em 01 de outubro de 1998, Lei Complementar n 050, intitulada Lei Orgânica dos Profissionais da Educação Básica (LOPEB).

Essa estrutura de carreira se embasa em dois componentes principais Classes e Níveis - e suas respectivas remunerações estabelecidas a partir de coeficientes diferenciados. Na linha horizontal, representadas pelas letras, estão estabelecidas as classes, estruturadas segundo os graus de formação exigidos para o provimento do cargo. Classe A: habilitação específica de nível médio-magistério; Classe B: habilitação específica de grau superior, em nível de graduação, representada por licenciatura; Classe C: habilitação específica de grau superior, em nível de graduação, representada por licenciatura, com especialização; Classe D: habilitação específica de grau superior, em nível de graduação, representada por licenciatura plena, com curso de mestrado na área de educação relacionado com sua habilitação; Classe E: habilitação específica de grau superior, em nível de graduação, representada por licenciatura plena, com curso de doutorado na área de educação relacionado com sua habilitação.

Cada classe desdobra-se em níveis, indicados por algarismos arábicos de 01 a 12, que constituem a linha vertical de progressão. $\mathrm{O}$ direito à progressão na carreira dá-se a cada três anos. Registra-se que o texto da Lei $n^{\circ} 050 / 98$ sofreu alterações referentes às classes e níveis, a partir da Lei Complementar n ${ }^{\circ}$ 206/04. Além de ampliar os níveis de 09 para 12, dividiu-se a Classe D, criando uma nova classe (Classe E), correspondente ao nível de formação de doutorado. Veja os QUADROS abaixo:

QUADRO 1

Classes e Coeficientes

\begin{tabular}{c|c|c|c}
\hline \multicolumn{2}{c|}{1998 ATÉ 2004} & \multicolumn{2}{c}{ A PARTIR DE 2005 } \\
\hline \multirow{2}{*}{ CLASSES } & COEFICIENTES & CLASSES & COEFICIENTES \\
\hline A & 1 & A & 1,00 \\
B & 1,5 & B & 1,50 \\
C & 1,7 & C & 1,70 \\
D & 1,85 & D & 2,02 \\
- & - & E & 2,30 \\
\hline
\end{tabular}

Fonte: Leis Complementares - 050/98 e 206/04. 


\section{QUADRO 2}

Níveis e Coeficientes

\begin{tabular}{c|c|c|c}
\hline \multicolumn{2}{c|}{1998 ATÉ 2004 } & \multicolumn{2}{c}{ A PARTIR DE 2005 } \\
\hline \multirow{2}{*}{ NÍVEIS } & COEFICIENTES & NÍVEIS & COEFICIENTES \\
\hline 1 & 1 & 1 & 1,00 \\
2 & 1,04 & 2 & 1,04 \\
3 & 1,085 & 3 & 1,085 \\
4 & 1,135 & 4 & 1,135 \\
5 & 1,19 & 5 & 1,19 \\
6 & 1,25 & 6 & 1,25 \\
7 & 1,32 & 7 & 1,32 \\
8 & 1,41 & 8 & 1,41 \\
9 & 1,5 & 9 & 1,50 \\
10 & - & 10 & 1,53 \\
11 & - & 11 & 1,56 \\
12 & & 12 & 1,59 \\
\hline
\end{tabular}

Fonte: Leis Complementares - 050/98 e 206/04.

A Lei Complementar $n^{\circ} 206$, de 29 de dezembro de 2004, instituiu as alterações sobre a Lei Complementar $n^{\circ} 050 / 98$, conforme demonstram os QUADROS acima, referentes às classes e aos níveis e seus respectivos coeficientes. Além disso, em seu artigo 24, determinou que seus efeitos financeiros surtissem efeitos a partir de janeiro de 2005.

Os efeitos dos coeficientes sobre a remuneração docente, a cada três anos trabalhados, leva à progressão de um nível para o outro. O GRAF. 1 , a seguir, demonstra as diferenças - em pontos percentuais - entre as remunerações de dois níveis sucessivos:

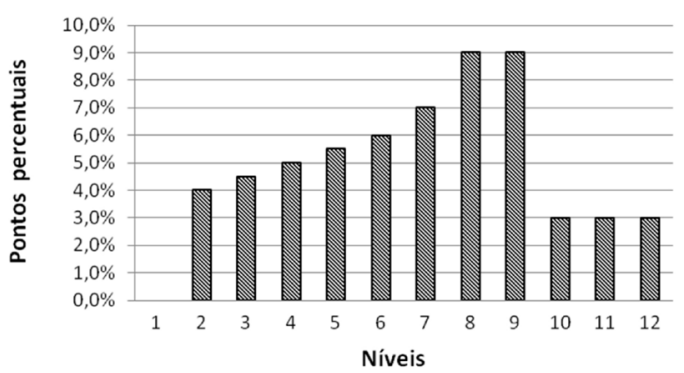

GRÁFICO 1 - Incrementos entre os níveis.

Fonte: Leis Complementares - 050/98 e 206/04. 
Como demonstra o GRAF. 1, passando do Nível 1 para o Nível 2, o subsídio recebe um incremento de 4,0\%; do Nível 2 para o Nível 3, de 4,5\%; do Nível 3 para o Nível 4, de 5,0\% e, assim, sucessivamente até do Nível 11 para o Nível 12, com 3,0\% de incremento. Esses incrementos - pontos percentuais - são sempre em relação ao subsídio do Nível 1 e considerando-se a mesma Classe. Nota-se que os maiores incrementos são quando se passa do Nível 7 para o Nível 8 e deste para o Nível 9, ambos iguais a 9,0\%. A partir da mudança para o Nível 10 e até o último Nível os incrementos devido ao tempo são todos iguais a 3,0\%, portanto os menores incrementos de toda a carreira.

\section{A evolução da remuneração a partir da carreira}

O artigo 44 da Lei Complementar n 050/98 definiu, também, o sistema remuneratório dos Profissionais da Educação Básica em forma de subsídio fixado em parcela única, vedado o acréscimo de qualquer gratificação, adicional, abono, prêmio, verba de representação ou qualquer espécie remuneratória, devendo ser revisto, obrigatoriamente, a cada 12 (doze) meses. O artigo 45 dessa lei instituiu o piso salarial para uma jornada de trabalho de 30 (trinta) horas semanais, abaixo da qual não haverá qualquer subsídio.

A TAB. 1 demonstra a evolução do subsídio de acordo com a estrutura de carreira aprovada e de fato implementada para o quadro de professores (as) efetivos(as) aprovados(as) em concurso público e empossados(as).

TABELA I

Classe A: Nível Médio - 1,0

Subsídios praticados

\begin{tabular}{c|c|c|c|c}
\hline & \multicolumn{4}{|c}{ SUBSIDIOS } \\
\hline \multirow{2}{*}{ NIVEIS } & $\mathbf{1 9 9 8}$ & $\mathbf{2 0 0 5}$ & $\mathbf{2 0 0 7}$ & $\mathbf{2 0 1 0}$ \\
& & & 788,25 & $1.135,16$ \\
2 & 465,00 & 648,77 & 819,78 & $1.180,57$ \\
3 & 483,60 & 674,72 & 855,25 & $1.231,65$ \\
4 & 504,53 & 703,92 & 894,66 & $1.288,41$ \\
5 & 527,78 & 736,35 & 938,02 & $1.350,84$ \\
\hline
\end{tabular}




\begin{tabular}{c|c|c|c|c}
\hline & \multicolumn{4}{|c}{ SUBSIDIOS } \\
\hline \multirow{2}{*}{ NIVEIS } & $\mathbf{1 9 9 8}$ & $\mathbf{2 0 0 5}$ & $\mathbf{2 0 0 7}$ & $\mathbf{2 0 1 0}$ \\
& 581,25 & 810,96 & 985,31 & $1.418,95$ \\
$\mathbf{7}$ & 613,80 & 856,38 & $1.040,49$ & $1.498,41$ \\
8 & 655,65 & 914,77 & $1.111,43$ & $1.600,58$ \\
9 & 697,5 & 973,16 & $1.182,38$ & $1.702,74$ \\
10 & - & 992,62 & $1.206,02$ & $1.736,79$ \\
11 & - & $1.012,08$ & $1.229,67$ & $1.770,85$ \\
12 & - & $1.031,54$ & $1.253,32$ & $1.804,90$ \\
\hline
\end{tabular}

Fonte: SECRETARIA DE ESTADO DE EDUCAÇÃO/MT.

A Classe A, referente ao nível médio, possui coeficiente 1,0 porque é a classe inicial, servindo como base para as outras classes. Por exemplo, os subsídios da Classe B - mantido o mesmo nível - são obtidos a partir da Classe A, aplicando-se o coeficiente 1,5. Conforme o Relatório NG39 SEAP/SAD 05/2010, em outubro de 2009, a Classe A possuía 176 docentes enquadrados na carreira, enquanto as Classes B e C possuíam 3.206 e 6.386, respectivamente. Nos Níveis 1 e 2 da Classe A não havia docentes enquadrados, enquanto a maioria estava nos Níveis 7 e 8 . A TAB. 2, abaixo, refere-se à Classe $\mathrm{B}$, representada pelos profissionais com a titulação de graduados, cuja remuneração tem como base o coeficiente 1,5. Ela demonstra os valores praticados nos anos de 1998, data de aprovação da Lei, do ano de 2004, em que se deu a ampliação dos níveis - representados pelos algarismos dez, onze e doze -, e dos anos 2007 e 2010.

TABELA 2

Classe B: Graduação - 1,5

Subsídios praticados

\begin{tabular}{c|c|c|c|c}
\hline & \multicolumn{4}{|c}{ SUBSIDIOS } \\
\hline \multirow{2}{*}{ NIVEIS } & $\mathbf{1 9 9 8}$ & $\mathbf{2 0 0 5}$ & $\mathbf{2 0 0 7}$ & $\mathbf{2 0 1 0}$ \\
& & & & \\
1 & 697,50 & 973,16 & $1.182,38$ & $1.702,74$ \\
2 & 725,40 & 1012,08 & $1.229,67$ & $1.770,86$ \\
3 & 756,79 & 1055,87 & $1.282,88$ & $1.847,48$ \\
\hline
\end{tabular}




\begin{tabular}{c|c|c|c|c}
\hline \multirow{2}{*}{ NIVEIS } & \multicolumn{4}{|c}{ SUBSIDIOS } \\
\hline & $\mathbf{1 9 9 8}$ & $\mathbf{2 0 0 5}$ & $\mathbf{2 0 0 7}$ & $\mathbf{2 0 1 0}$ \\
4 & 791,66 & 1104,53 & $1.341,99$ & $1.932,62$ \\
5 & 830,03 & 1158,05 & $1.407,03$ & $2.026,26$ \\
6 & 871,88 & 1216,44 & $1.477,97$ & $2.128,43$ \\
7 & 920,70 & 1284,56 & $1.560,74$ & $2.247,62$ \\
8 & 983,48 & 1372,15 & $1.667,15$ & $2.400,87$ \\
9 & $1.046,25$ & 1459,73 & $1.773,57$ & $2.554,11$ \\
10 & - & 1488,93 & $1.809,03$ & $2.605,19$ \\
11 & - & 1518,12 & $1.844,51$ & $2.656,28$ \\
12 & - & 1547,32 & $1.879,98$ & $2.707,35$ \\
\hline
\end{tabular}

Fonte: SECRETARIA DE ESTADO DE EDUCAÇÃO/MT.

Para cada classe e em seu respectivo nível é praticado um valor remuneratório diferenciado, calculado a partir dos respectivos coeficientes. Cabe registrar que os valores estabelecidos podem ser comprovados a partir de holerites mensais extraídos da folha de pagamento, em nome do (a) professor (a), com os respectivos números de matrícula e dados documentais, como os números de registro de identidade e CPF.

A TAB. 3 trata dos subsídios dos docentes enquadrados na Classe C: habilitação específica de graduação, representada por licenciatura, com especialização, cujo coeficiente está estipulado em 1,7.

TABELA 3

Classe C: Especialização - 1,7

Subsídios Praticados

\begin{tabular}{c|c|c|c|c}
\hline & \multicolumn{4}{|c}{ SUBSIDIOS } \\
\hline \multirow{2}{*}{ NIVEIS } & $\mathbf{1 9 9 8}$ & $\mathbf{2 0 0 5}$ & $\mathbf{2 0 0 7}$ & $\mathbf{2 0 1 0}$ \\
& & & & \\
1 & 790,50 & 1102,91 & $1.340,03$ & $1.929,77$ \\
2 & 822,12 & 1147,03 & $1.393,63$ & $2.006,97$ \\
4 & 857,69 & 1196,66 & $1.453,93$ & $2.093,81$ \\
5 & 897,22 & 1251,8 & $1.520,92$ & $2.190,30$ \\
6 & 940,70 & 1312,46 & $1.594,63$ & $2.296,43$ \\
& 988,13 & 1378,64 & $1.675,03$ & $2.412,22$ \\
\hline
\end{tabular}




\begin{tabular}{c|c|c|c|c}
\hline & \multicolumn{4}{|c}{ SUBSIDIOS } \\
\hline \multirow{2}{*}{ NIVEIS } & $\mathbf{1 9 9 8}$ & $\mathbf{2 0 0 5}$ & $\mathbf{2 0 0 7}$ & $\mathbf{2 0 1 0}$ \\
& & 1455,84 & $1.768,83$ & $2.547,30$ \\
7 & $1.043,46$ & 1555,1 & $1.889,43$ & $2.720,99$ \\
9 & $1.114,61$ & 1654,36 & $2.010,05$ & $2.894,66$ \\
10 & $1.185,75$ & 1687,45 & $2.050,23$ & $2.952,54$ \\
11 & & 1720,54 & $2.090,44$ & $3.010,45$ \\
12 & & 1753,63 & $2.130,64$ & $3.068,33$ \\
\hline
\end{tabular}

Registra, ainda, que a natureza do subsídio, conforme já citado, não permite gratificações diferenciadas. O cargo de professor (a), além da função de docência, pode compreender, ainda, as funções de coordenação pedagógica e de direção de unidade escolar. A remuneração, para os casos citados, varia de acordo com a função. Para a coordenação pedagógica, o (a) docente recebe uma remuneração, em forma de subsídio, para 40 (quarenta) horas de trabalho semanais. Para a direção escolar, uma remuneração que varia de acordo com o tamanho da unidade escolar, os turnos de funcionamento e o número de alunos, regulamentados anualmente a partir de portarias emitidas pela Secretaria de Estado de Educação. Essas funções são classificadas como de dedicação exclusiva.

Os GRAF. 2 a 5 permitem acompanhar a evolução salarial das classes A, B e C, em seus respectivos níveis, exibindo os subsídios de 4 (quatro) anos. Analisando-os conjuntamente, pode-se observar que, apesar das mudanças em termos de valores, alguns aspectos se mantêm: a) do primeiro ao último nível, mas fixando uma classe, os subsídios são crescentes; b) da Classe A à Classe $C$, mas fixando um nível, os subsídios são crescentes; c) os incrementos nos níveis, fixando uma classe, são maiores, até se atingir o Nível 9, ou seja, os subsídios aumentam mais rapidamente até este nível. 
$\mathrm{QA} \otimes \mathrm{B} \cap \mathrm{D}$

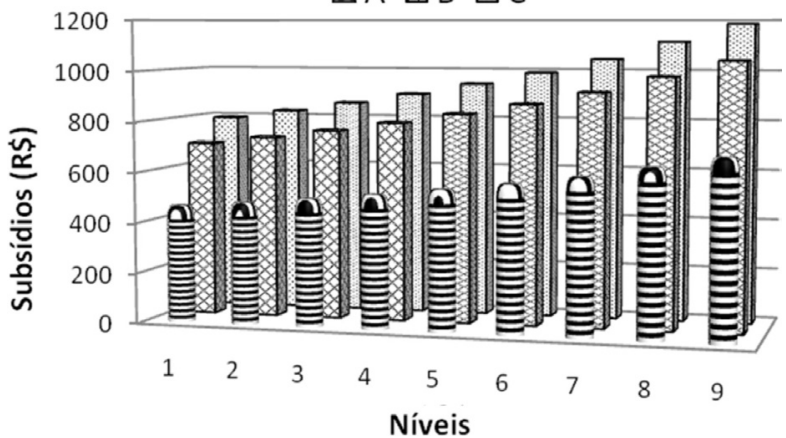

GRÁFICO 2 - Remuneração de Professores: Classes A, B e C - 1998. Fonte: SECRETARIA DE ESTADO DE EDUCAÇÃO/MT.

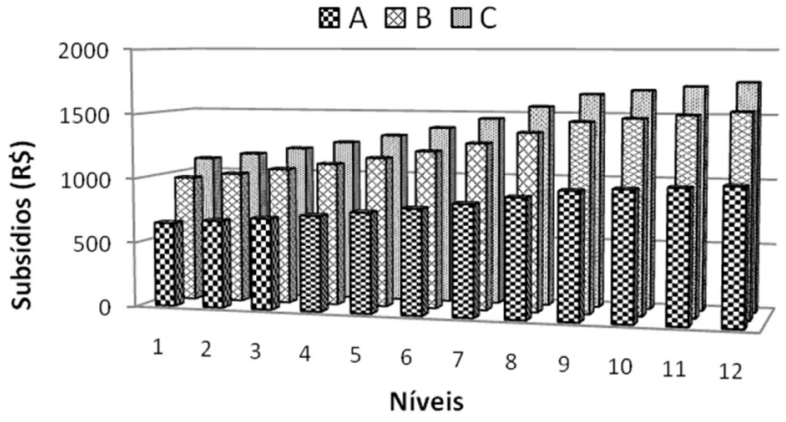

GRÁFICO 3 - Remuneração de Professores: Classes A, B e C - 2005. Fonte: SECRETARIA DE ESTADO DE EDUCAÇÃO/MT.

$\mathrm{NA}$ 囷B $\square \mathrm{C}$

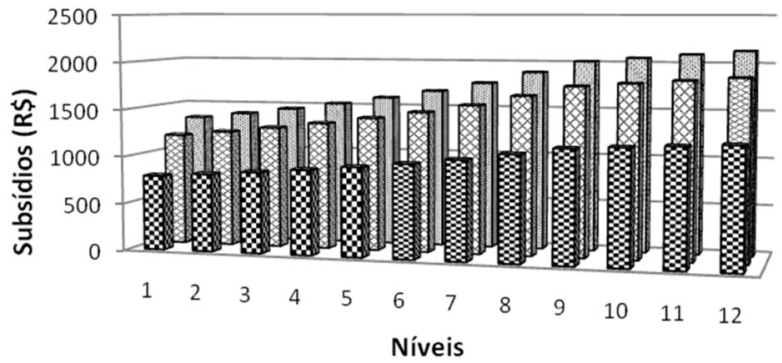

GRÁFICO 4 - Remuneração de Professores: Classes A, B e C - 2007. Fonte: SECRETARIA DE ESTADO DE EDUCAÇÃO/MT. 


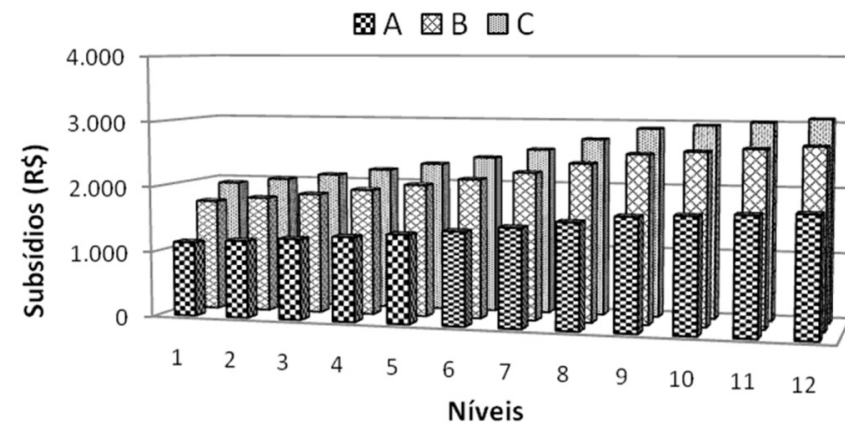

GRÁFICO 5 - Remuneração de Professores: Classes A, B e C - 2010. Fonte: SECRETARIA DE ESTADO DE EDUCAÇÃO/MT.

Percebe-se que a carreira docente no Estado de Mato Grosso, no que tange à remuneração, possui em sua estrutura, organizada em classes e níveis com seus respectivos coeficientes, bases suficientes para viabilizar uma efetiva política de valorização dos profissionais da educação. $\mathrm{O}$ subsídio base, do nível inicial em cada classe, é a referência para a evolução da remuneração no decorrer do tempo trabalhado, representado pelos níveis, a partir da aplicação dos coeficientes estabelecidos.

A evolução, em termos de remuneração, também é possível a partir da ascensão de uma classe para outra, mediante a comprovação da aquisição de novos títulos de formação além da graduação - especialização, mestrado e doutorado - e o respectivo enquadramento dentro da própria carreira, dispensando a realização de um novo concurso público.

Cabe registrar, entretanto, que a carreira docente, no Estado de Mato Grosso, ainda não permite a real valorização de seus profissionais em razão dos valores estabelecidos como subsídios iniciais para o Nível 1. Essa melhoria, em termos remuneratórios, ainda carece de uma política efetiva de financiamento da educação, especialmente no que tange à operação de um custo-aluno-qualidade capaz de garantir um piso salarial mais substancial e atraente para os profissionais da área. $O$ piso remuneratório dos profissionais da educação é inferior aos pisos de diversas outras profissões. 


\section{Conclusão}

A lei de carreira dos profissionais da educação básica é um dos principais elementos geradores da valorização docente. No Estado de Mato Grosso, esta lei foi criada em 1998 e, de fato, tem sido implementada para todo o quadro efetivo, ingressado na carreira a partir de concurso público. A transposição para novos níveis, de acordo com o tempo, tem sido implementada, o que tem garantido a evolução dos subsídios (remuneração).

Entretanto, a recomposição do quadro de profissionais ativos mediante concurso público e ingresso na carreira não tem sido efetivada na mesma proporção das aposentadorias dos profissionais, da ampliação de matrículas de alunos na esfera estadual e do surgimento de aulas livres e, consequentemente, de novas vagas para concurso público.

Essas aulas livres têm sido ocupadas por profissionais não concursados, denominados interinos, não beneficiados pelas vantagens da Lei Complementar $n^{\circ}$ 050/98. Essa restrição de vantagens se dá especialmente em termos de jornada de trabalho. O professor efetivo é remunerado por 30 horas semanais, sendo 10 horas de trabalho pedagógico, ao passo que o professor interino é contratado precariamente, remunerado apenas pelas horas de trabalho, em sala de aula, diretamente com alunos.

Esse fator tem limitado a valorização desses profissionais no Estado. Além desse aspecto, a baixa remuneração tem levado à multiplicação da jornada de trabalho, não só do quadro de contratados, mas também do quadro efetivo, o que tem comprometido tanto a saúde desses profissionais, quanto a qualidade do processo educacional. 


\section{Referências}

MATO GROSSO. Lei Complementar n. 50, de 01 out. 1998. Dispõe sobre a Carreira dos Profissionais da Educação Básica de Mato Grosso. Diário Oficial, Cuiabá, 01 out. 1998. Disponível em:<http://www. al.mt.gov.br>.

MATO GROSSO. Lei Complementar n. 206, de 29 dez. 2004. Dispõe sobre alterações na Lei Complementar n. 50, de 01 de outubro de 1998. Diário Oficial, Cuiabá, 29 dez. 2004. Disponível em:<http://www. al.mt.gov.br>.

MATO GROSSO. Lei Complementar n. 277, de 06 set. 2007. Fixa o subsídio dos Profissionais da Educação Básica do Poder Executivo Estadual e da outras providências. Diário Oficial, Cuiabá, 06 set. 2007. Disponível em:<http://www.al.mt.gov.br>.

MATO GROSSO. Lei Complementar n. 388, de 31 mar. 2010. Fixa o subsídio dos Profissionais da Educação Básica e o vencimento dos Especialistas de Educação do Poder Executivo Estadual e dá outras providências. Diário Oficial, Cuiabá, 31 mar. 2010. Disponível em:<http://www.al.mt.gov.br>.

MATO GROSSO. Secretaria de Estado de Educação. Secretaria Adjunta de Gestão de Políticas Institucionais de Pessoal. Indicadores de gestão de pessoas: 2003-2009. Cópia de transparências. 


\title{
Basic education teachers remuneration in Mato Grosso: its evolution in the context of law career and challenges for recovery education professional
}

\begin{abstract}
The study aims to locate and analyze the configuration and evolution of the Basic Education teacher remuneration in the State of Mato Grosso. Based on the legal career framework and remuneration will be demonstrated to policy developments in the state valuation of such professionals. The remuneration itself will be presented in its originality in the period 1996 to 2010 from the teacher training: within the High School, Graduation and Postgraduation represented by class career, working hours and work experience in the teaching function, considering the beginning and end of their careers. Complementary Law 050 from October 1st 1998 establishing the careers of Basic Education Professionals in the State. The monitoring of the evolution of remuneration defined as salary, set in a single plot, practiced according to the classes and levels, structural elements of the career, to discover the structure and dynamics of the teaching profession, which in addition to teachers also covers other professionals working in Basic Education. The study presented here refers to the professorship and shows from charts, tables and graphs the coefficients and the respective values of allowances (remuneration) is operated for each class and level, according to the time worked. The initial level is the basis for definition of Professional Floor Wage and serves as a parameter for the other inserted incrementally over the during the teaching career. The reflections are situated in the context of the financing of basic education and take into account the policy defined in the FUNDEF and
\end{abstract}


FUNDEB. Wage developments will be reflected from both normative legal instruments of the funding policy of education and National Professional Floor Wage, the Organic Law of Education Professionals in the State of Mato Grosso.

Keywords: career; education; financing; remuneration; valuation; working hours.

Recebido: 18/01/2012

Aprovado: 19/06/2012 